\title{
Utility of BRAF protein overexpression in predicting the metastasis potential of papillary thyroid carcinoma
}

\author{
LU FENG ${ }^{1}$, MEI LI ${ }^{1}$, QIU-PING ZHANG ${ }^{2}$, ZHENG-AI PIAO ${ }^{3}$, ZHAO-HUI WANG $^{1}$ and SHEN LV ${ }^{1}$ \\ ${ }^{1}$ Laboratory Center, The Second Affiliated Hospital of Dalian Medical University, Liaoning 116027; \\ ${ }^{2}$ Department of Pathology, The First Affiliated Hospital of Dalian Medical University, Liaoning 116011; \\ ${ }^{3}$ Department of Pathology, The Third People's Hospital of Dalian, Liaoning 116033, P.R. China
}

Received August 11, 2010; Accepted November 15, 2010

DOI: $10.3892 / \mathrm{ol} .2010 .219$

\begin{abstract}
V-raf murine sarcoma viral oncogene homolog B1 (BRAF) is a significant member of the MAPK pathway, the point mutation (V600E) of which is a common genetic event in papillary thyroid carcinoma (PTC). Investigators showed that the variations in BRAF expression levels were independent of the V600E mutation. These variations were involved in the pathogenesis of thyroid carcinomas. This study evaluated the feasibility of BRAF, proliferating cell nuclear antigen (PCNA) and hMSH2 as markers for the prediction of the metastatic potential of PTC. Using immunohistochemistry, the expression of BRAF, PCNA and hMSH2 proteins was studied in 70 PTC and 29 nodular goiter (NG) tissues. The results indicated that i) the positive rate of BRAF, PCNA and hMSH2 expression in PTCs was significantly higher than that in NGs $(\mathrm{P}=0.000, \mathrm{P}=0.000$ and $\mathrm{P}=0.003$, respectively), ii) the positive rate of BRAF expression in the lymph node metastasis (LNM) group was significantly higher than that in the non-LNM group $(\mathrm{P}=0.019)$, iii) the age at diagnosis of PTC patients with LNM was significantly older compared to that without $\mathrm{LNM}(\mathrm{P}=0.021)$ and iv) the positive rate of BRAF expression significantly correlated with that of PCNA and hMSH2 expression ( $\mathrm{P}=0.000$ and $\mathrm{P}=0.019$, respectively). In conclusion, BRAF, PCNA and hMSH2 overexpression appeared to be molecular events of PTC carcinogenesis. Older patients with BRAF overexpression appear to be a high-risk group for PTC metastasis. Detection of BRAF expression is likely to aid in the prediction of the metastatic potential of the carcinoma.
\end{abstract}

Correspondence to: Professor Shen Lv, Laboratory Center, The Second Affiliated Hospital of Dalian Medical University, Dalian, Liaoning 116027, P.R. China

E-mail: lushen1956@yahoo.com.cn

Key words: papillary thyroid carcinoma, V-raf murine sarcoma viral oncogene homolog B1, proliferating cell nuclear antigen, hMSH2, immunohistochemistry

\section{Introduction}

Papillary thyroid carcinoma (PTC) is the most prominent malignancy of the thyroid gland, accounting for more than $80 \%$ of all thyroid cancers. The majority of PTC patients have a favorable prognosis with the appropriate treatment, but a poor prognosis, such as recurrence, metastasis or even death, is noted in other patients. Once metastasis occurs, the therapeutic effect of PTC shows a marked decrease. Therefore, agents are required to predict the metastatic potential of the carcinoma. Such agents may be utilized as clinical guidance for the further treatment and post-operative management of patients. It was reported that PTC exhibiting infiltration of adjacent tissues was likely to recur and lead to a poor prognosis (1). These malignant biological behaviors result from aberrant genetic alterations and protein expression in cancer cells. Thus, advanced understanding of the molecular events associated with PTC pathogenesis may lead to the confirmation of more effective PTC prognostic markers, which would aid in the prediction of the metastatic potential of the carcinoma.

V-raf murine sarcoma viral oncogene homolog B1 (BRAF) gene encodes a serine/threonine protein kinase, BRAF protein, as a key member of the RAS/RAF/MEK/ ERK/MAPK pathway (2). Previous studies showed that the BRAF mutation (V600E) played a role in the molecular carcinogenesis of PTC by aberrant activation of the MAPK pathway $(3,4)$, whereas the association of BRAF mutation with prognosis of the metastatic potential was controversial (5-8). Certain studies showed that the variations of BRAF protein expression levels were independent of the V600E mutation in thyroid carcinomas and the carcinomas were induced by mutation or protein overexpression of the gene, respectively. (9) To the best of our knowledge, little information is available on BRAF expression with regard to the metastatic potential of PTC. Unrestrained cell proliferation is a fundamental biological behavior of carcinomas, which may be regarded as a prognostic marker. Proliferating cell nuclear antigen (PCNA) was originally identified as a protein expressing in the nuclei during the DNA synthesis phase and is deemed to be a marker for cell proliferation (10). The overexpression of PCNA was observed in various malignant carcinomas, since carcinomas always exhibit uncontrolled proliferation $(11,12)$. Cvejic et al 

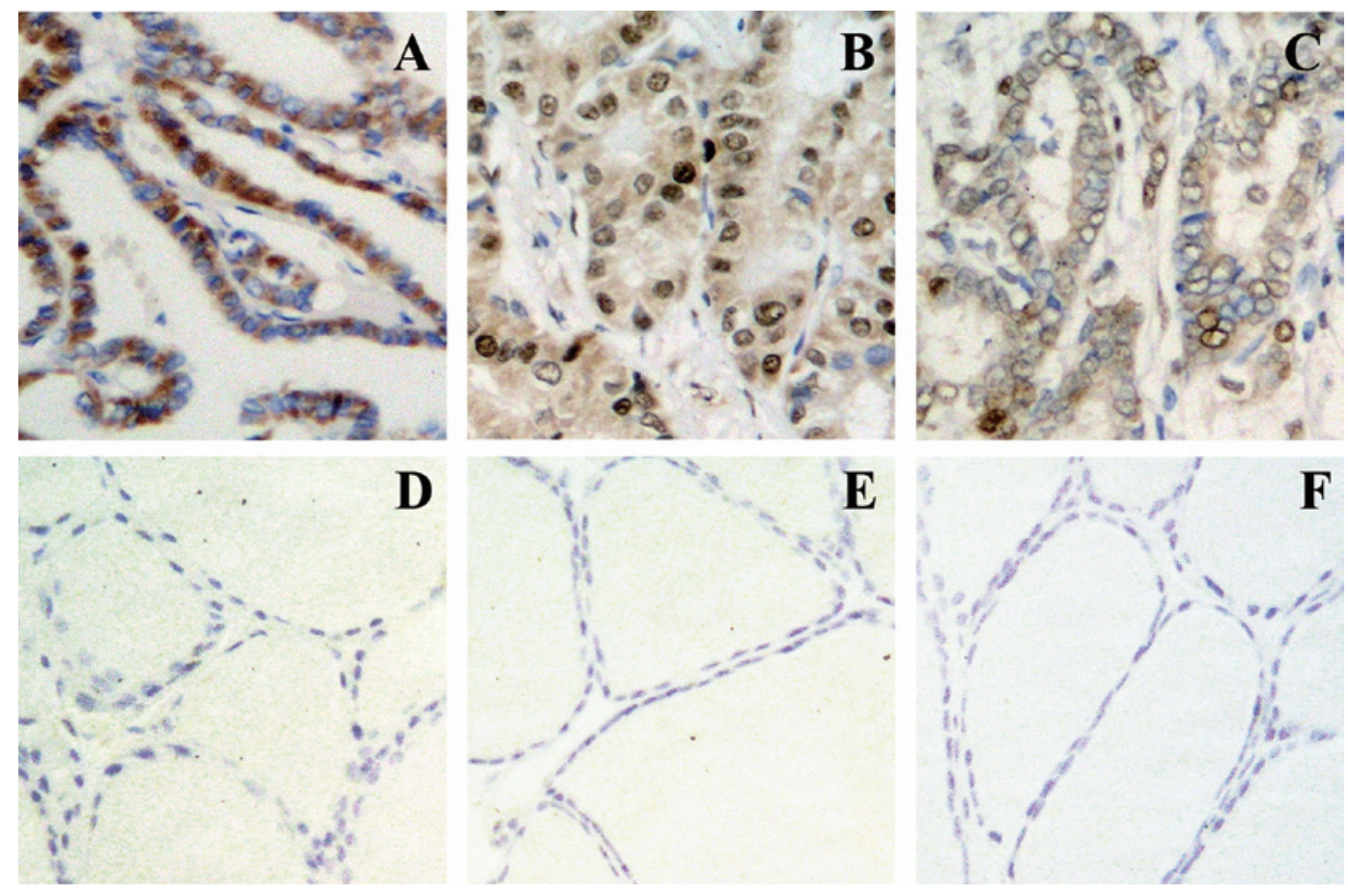

Figure 1. Immunohistochemical profiling of the positive expression of (A, B and C) BRAF, PCNA and hMSH2 protein in papillary thyroid carcinomas and (D, E and F) the negative expression in NGs. Magnification, $\mathrm{x} 200$.

reported that PCNA expression was significantly up-regulated according to the increasing malignancy of thyroid carcinomas (13). The association of the proliferation status with the metastatic potential of PTC required investigation.

The mismatch repair (MMR) system is primarily responsible for correcting DNA base-base mismatches and base insertion/deletion error during DNA replication, thereby preventing mutagenesis and maintaining genomic stability (14). The expression of MMR genes generally maintains a high level in labile or cancer cells that exhibit unrestrained proliferation due to the increasing rate of base mismatching during the replication process of cell proliferation. As stable cells, the thyroid cells are inactive with only minimal replicative activity. Base-base mismatches in the cells rarely occur and the MMR gene expression often maintains a low level in normal thyroid cells. The hMSH2 gene is the first identified MMR gene. Deficiency of the hMSH2 gene results in the incidence of numerous carcinomas (15). On the other hand, the protein overexpression of the gene was detected in certain carcinomas (16). However, the function status and expression level of hMSH2 in PTC remains controversial.

In this study, the protein expression of BRAF, PCNA and hMSH2 in PTC, with and without lymph node metastasis was detected. Moreover, these agents served as novel markers in the prediction of the metastatic potential of PTC.

\section{Materials and methods}

Patients and tissue samples. A total of 70 PTC and 29 nodular goiter (NG) tissues were used in this study. The tissues were obtained from patients who underwent surgery between 2006 and 2007 at certain hospitals in Dalian, China. Among the 70 PTC tissues, 21 cases presented with lymph node metastasis (LNM group) and 49 cases were without lymph node metastasis (non-LNM group); 12 male and 58 female cases with a mean \pm SD age at diagnosis of $50.0 \pm 14.4$ years (range 19-87). The formalin-fixed paraffin-embedded tissue sections were used following the approval of the Ethics Committee of Dalian Medical University, after informed consent was obtained from the patients, and the histological diagnoses of the samples were independently established according to the World Health Organization classification by two certified pathologists.

Immunohistochemistry. The specimens were fixed in $10 \%$ neutral-buffered formalin and embedded in serial 3-mm paraffin blocks. Sections $(4-\mu \mathrm{m})$ were prepared for immunohistochemistry (IHC). Monoclonal antibodies were used as follows: BRAF: F-7, 1:500 dilution (Santa Cruz); hMSH2: FE 11, 1:250 dilution (Zymed Lab Inc., San Francisco, CA, USA); and PCNA: PC10, 1:200 dilution (NeoMarkers). IHC was performed according to the manufacturer's instructions. Briefly, the slides were deparaffinized with xylene and rehydrated through graded ethanol to distilled water. Endogenous peroxidase was quenched in $3 \% \mathrm{H}_{2} \mathrm{O}_{2}$. The sections were rinsed with phosphate-buffered saline (PBS), processed for antigen retrieval with $10 \mathrm{mM}$ citrate buffer ( $\mathrm{pH}$ 6.0) and allowed to cool naturally at room temperature. Then, 10\% normal horse serum was used to block nonspecific binding. The sections were incubated with primary antibodies at $4^{\circ} \mathrm{C}$ overnight, secondary antibodies at $37^{\circ} \mathrm{C}$ for $30 \mathrm{~min}$ and streptavidin peroxidase at $37^{\circ} \mathrm{C}$ for $30 \mathrm{~min}$. The reaction was visualized using 3,3'-diaminobenzidinetetrahydrochloride (DAB) as chromogen and was stopped by the addition of water after $5 \mathrm{~min}$. The sections were counterstained with hematoxylin. Primary antibodies 
Table I. Variations in BRAF, PCNA and hMSH2 expression between PTC and NG.

\begin{tabular}{|c|c|c|c|c|c|c|c|}
\hline & \multirow[t]{2}{*}{ No. } & \multicolumn{2}{|c|}{ BRAF } & \multicolumn{2}{|c|}{ PCNA } & \multicolumn{2}{|c|}{ hMSH2 } \\
\hline & & + & P-value & + & P-value & + & P-value \\
\hline PTC, no. (\%) & 70 & $42(60.0)$ & 0.000 & $28(40.0)$ & 0.000 & $18(25.7)$ & 0.003 \\
\hline NG, no. (\%) & 29 & $0(0.0)$ & 0.000 & $0(0.0)$ & 0.000 & $0(0.0)$ & 0.003 \\
\hline
\end{tabular}

BRAF, V-raf murine sarcoma viral oncogene homolog B1; PCNA, proliferating cell nuclear antigen; PTC, papillary thyroid carcinoma; NG, nodular goiter; + , positive expression.

Table II. Correlations of BRAF, PCNA and hMSH2 expression with the clinicopathological characteristics of PTC patients.

\begin{tabular}{|c|c|c|c|c|c|c|c|}
\hline & \multirow[b]{2}{*}{ No. } & \multicolumn{2}{|c|}{ BRAF } & \multicolumn{2}{|c|}{ PCNA } & \multicolumn{2}{|c|}{ hMSH2 } \\
\hline & & + & P-value & + & P-value & + & P-value \\
\hline Age (no.) & 70 & 24 & NS & 28 & NS & 18 & NS \\
\hline Mean \pm SD & $50.0 \pm 14.4$ & $51.4 \pm 16.1$ & & $48.3 \pm 18.3$ & & $51.4 \pm 16.9$ & \\
\hline Gender, no. (\%) & & & NS & & NS & & NS \\
\hline Male & 12 & $6(50.0)$ & & $5(41.7)$ & & $2(16.7)$ & \\
\hline Female & 58 & $36(62.1)$ & & $23(39.7)$ & & $16(27.6)$ & \\
\hline LNM, no. (\%) & & & 0.019 & & NS & & NS \\
\hline Present & 21 & $17(81.0)$ & & $10(47.6)$ & & $5(23.8)$ & \\
\hline Absent & 49 & $25(51.0)$ & & $18(36.7)$ & & $13(26.5)$ & \\
\hline
\end{tabular}

BRAF, V-raf murine sarcoma viral oncogene homolog B1; PCNA, proliferating cell nuclear antigen; PTC, papillary thyroid carcinoma; LNM, lymph node metastasis; +, positive expression; NS, not significant $(\mathrm{P}>0.05)$.

Table III. Correlations of BRAF expression with hMSH2 and PCNA expression.

\begin{tabular}{|c|c|c|c|c|c|c|}
\hline & \multicolumn{3}{|c|}{ PCNA } & \multicolumn{3}{|c|}{ hMSH2 } \\
\hline & + & - & P-value & + & - & P-value \\
\hline BRAF & & & 0.000 & & & 0.019 \\
\hline Positive & 24 & 18 & & 15 & 27 & \\
\hline Negative & 4 & 24 & & 3 & 25 & \\
\hline
\end{tabular}

BRAF, V-raf murine sarcoma viral oncogene homolog B1; PCNA, proliferating cell nuclear antigen; +, positive expression; -, negative expression.

were replaced by PBS in the negative control. The detailed protocol of IHC was performed as described by Li et al (16).

Assessment of staining and statistical data. The immunoreactivity of BRAF and hMSH2 was evaluated as: $(-),<10 \%$ of parenchymal cells showed positive immunoreactivity; and $(+), \geq 10 \%$ showed positive immunoreactivity. The immunoreactivity of PCNA was evaluated as: $(-),<20 \%$ of parenchymal cells showed positive immunoreactivity; and $(+), \geq 20 \%$ showed positive immunoreactivity. The staining results were independently evaluated by two investigators.

Statistical comparisons of data were performed by Student's t-test, Chi-square test and Spearman's correlation analysis on SPSS 13.0 software. $\mathrm{P}<0.05$ was considered to be statistically significant.

\section{Results}

The immunoreactivity of BRAF was localized in the cytoplasm and those of PCNA and hMSH2 were localized in the nuclei (Fig. 1). Variations in the positive rates of BRAF, PCNA and hMSH2 expression between PTC and NG are shown in Table I. The positive rate of BRAF, PCNA and hMSH2 expression in PTCs was significantly higher than that in NGs $(\mathrm{P}=0.000$, $\mathrm{P}=0.000$ and $\mathrm{P}=0.003$, respectively). The immunoreactions of BRAF, PCNA and hMSH2 were not detected in NGs.

The correlations of BRAF, hMSH2 and PCNA expression with the clinicopathological characteristics of the PTC patients are shown in Table II. The positive rate of BRAF expression in the LNM group was significantly higher compared to that in the non-LNM group ( $\mathrm{P}=0.019)$. No correlation of hMSH2 and PCNA expression with the LNM status of the PTC patients was found.

The age at diagnosis of PTC patients with LNM $(56.0 \pm 14.7$ years) was found to be significantly older than that 
without LNM $(47.4 \pm 13.7$ years $)(\mathrm{P}=0.021)$. No correlation between the LNM status and gender of the PTC patients was noted.

Correlations of the BRAF expression with $\mathrm{hMSH} 2$ and PCNA expression is shown in Table III. The positive rate of BRAF expression significantly correlated with that of PCNA and hMSH2 expression ( $\mathrm{P}=0.000$ and $\mathrm{P}=0.019$, respectively).

\section{Discussion}

PTC derives from the follicular epithelium and is the most common subtype of thyroid cancer. Although many PTC patients have favorable outcomes, a poor, or even fatal, prognosis is noted in other patients. In general, elderly individuals often receive a worse prognosis. In our study, metastasis was correlated with age, indicating that metastasis was common in elderly patients.

Certain cancer cells that are able to survive the journey to another area of the body, metastasize. In other words, the potential for metastasis and prognosis of PTC patients, is determined by the biological behaviors of the carcinoma, the alterations of which rely on the variations of gene and dysfunction or the aberrant activation of vital proteins. Therefore, the aberrant expression of certain proteins may be a marker for malignant molecular events in cells, aiding in the prediction of biological behavior as a metastatic potential of PTC.

BRAF is a key activator of the MAPK pathway, which regulates cell survival, differentiation, apoptosis and proliferation. BRAF protein expresses with various levels according to the type of tissue. In this study, the positive rate of BRAF expression in PTC was significantly higher than that in NG. Similarly, among the PTC tissues, the positive rate was significantly higher in the LNM group than in the non-LNM group. Previous investigations suggested that the gene copy number gain involves one mechanism causing overexpression of the BRAF protein and is a new mechanism of BRAF activation in thyroid carcinomas (17). We hypothesize that the gene copy number gain occurs in the carcinogenesis of PTC, abnormally up-regulating the BRAF expression and deteriorating cell biological behaviors, leading to cell metastasis. It appears that the high level of BRAF expression was a particular phenotype feature of the metastatic potential in PTC. Furthermore, a method routinely used in numerous laboratories with simpler procedures than DNA mutation detection was used to identify the BRAF protein.

PCNA encodes an auxiliary protein of DNA polymerase $\delta$. PCNA expression in the S phase of the cell cycle is used to evaluate the proliferative activity of cells, whereas normal thyroid cells rarely proliferate as stable cells; thus, the PCNA expression maintains a low level. Certain studies showed that PCNA expression levels varied according to the heterogeneity of thyroid carcinomas (13). Our data showed that PCNA expression was higher in PTCs compared to that of NGs and was concomitant with the BRAF protein in PTC. Consequently, PTC cells with BRAF overexpression expressed proliferation.

hMSH2, a member of the MMR system, is responsible for genomic stability. Normal thyroid cells have been found to maintain a low level of hMSH2 expression, which was higher in thyroid carcinomas. In our study, the expression of
hMSH2 in PTC was markedly higher than that in NG and was concomitant with that of BRAF in PTC. Previous studies suggested that BRAF mutations were rarely attributable to the defection of hMSH2 in carcinomas (18). Nevertheless, as regards protein levels, an indirect relationship exists between BRAF and hMSH2. The correlation of BRAF to hMSH2 expression is shown by the fact that BRAF expression was up-regulated to carry out its molecular functions and induce thyroid cells to proliferate excessively, followed by the upregulation of hMSH2 expression in order to repair the increased mismatching errors during cell proliferation. Therefore, up-regulation of the hMSH2 expression may indicate the increasing proliferative capacity of cells.

In conclusion, BRAF, PCNA and hMSH2 overexpression was a molecular event of PTC tumorigenesis. Elderly patients with BRAF overexpression are a high-risk group for PTC metastasis. Our data on BRAF, PCNA and hMSH2 expression showed that BRAF activated the MAPK downstream pathway independently, inducing the unrestrained proliferation of cells with the presence of PCNA overexpression and increasing mismatched errors indirectly during frequent cell proliferation with the presence of hMSH2 overexpression. Although the specific mechanism for these relationships has yet to be elucidated, the detection of BRAF expression may aid in the prediction of the metastatic potential of PTC.

\section{Acknowledgements}

We thank Shihua Cheng and Liying Guo of the First Affiliated Hospital of Dalian Medical University for the sample collection and technical assistance.

\section{References}

1. Mai KT, Perkins DG, Yazdi HM, Commons AS, Thomas J and Meban S: Infiltrating papillary thyroid carcinoma: review of 134 cases of papillary carcinoma. Arch Pathol Lab Med 122: 166-171, 1998

2. Michaloglou C, Vredeveld LC, Mooi WJ and Peeper DS: BRAF (E600) in benign and malignant human tumours. Oncogene 27: 877-895, 2008.

3. Cohen Y, Xing M, Mambo E, Guo Z, Wu G, Trink B, Beller U, Westra WH, Ladenson PW and Sidransky D: BRAF mutation in papillary thyroid carcinoma. J Natl Cancer Inst 95: 625-627, 2003.

4. Dhomen $\mathrm{N}$ and Marais R: New insight into BRAF mutations in cancer. Curr Opin Genet Dev 17: 31-39, 2007.

5. Kebebew E, Weng J, Bauer J, Ranvier G, Clark OH, Duh QY, Shibru D, Bastian B and Griffin A: The prevalence and prognostic value of BRAF mutation in thyroid cancer. Ann Surg 246: 466-470, 2007

6. Liu RT, Chen YJ, Chou FF, Li CL, Wu WL, Tsai PC, Huang CC and Cheng JT: No correlation between BRAFV600E mutation and clinicopathological features of papillary thyroid carcinomas in Taiwan. Clin Endocrinol 63: 461-466, 2005.

7. Ito $\mathrm{Y}$, Yoshida H, Maruo R, et al: BRAF mutation in papillary thyroid carcinoma in a Japanese population: its lack of correlation with high-risk clinicopathological features and disease-free survival of patients. Endocr J 56: 89-97, 2009.

8. Lassalle S, Hofman V, Ilie M, Butori C, Bozec A, Santini J, Vielh P and Hofman P: Clinical impact of the detection of BRAF mutations in thyroid pathology: potential usefulness as diagnostic, prognostic and theragnostic applications. Curr Med Chem 17: 1839-1850, 2010.

9. Kondo T, Nakazawa T, Murata S, Kurebayashi J, Ezzat S, Asa SL and Katoh R: Enhanced B-Raf protein expression is independent of V600E mutant status in thyroid carcinomas. Hum Pathol 38: 1810-1818, 2007. 
10. Baserga R: Growth regulation of the PCNA gene. J Cell Sci 98: 433-436, 1991

11. Leonardi E, Girlando S, Serio G, Mauri FA, Perrone G, Scampini S, Dalla Palma P and Barbareschi M: PCNA and Ki67 expression in breast carcinoma: correlations with clinical and biological variables. J Clin Pathol 45: 416-419, 1992.

12. El-kott AF, El-baz MA and Mokhtar AA: Proliferating cell nuclear antigen (PCNA) overexpression and microvessel density predict survival in the urinary bladder carcinoma. Int Urol Nephrol 38: 237-242, 2006.

13. Cvejic D, Selemetjev S, Savin S, Paunovic I and Tatic S: Changes in the balance between proliferation and apoptosis during the progression of malignancy in thyroid tumours. Eur J Histochem 53: 65-71, 2009.

14. Jun SH, Kim TG and Ban C: DNA mismatch repair system. Classical and fresh roles. FEBS J 273: 1609-1619, 2006
15. Plevová $\mathrm{P}$, Krepelová A, Papezová M, et al: Immunohistochemical detection of the hMLH1 and hMSH2 proteins in hereditary nonpolyposis colon cancer and sporadic colon cancer. Neoplasma 51: 275-284, 2004.

16. Li M, Liu L, Wang Z, Wang L, Liu Z, Xu G and Lu S: Overexpression of hMSH2 and hMLH1 protein in certain gastric cancers and their surrounding mucosae. Oncol Rep 19: 401-406, 2008.

17. Ciampi R, Zhu Z and Nikiforov YE: BRAF copy number gains in thyroid tumors detected by fluorescence in situ hybridization. Endocr Pathol 16: 99-105, 2005.

18. Wang L, Cunningham JM, Winters JL, Guenther JC, French AJ, Boardman LA, Burgart LJ, McDonnell SK, Schaid DJ and Thibodeau SN: BRAF mutations in colon cancer are not likely attributable to defective DNA mismatch repair. Cancer Res 63: 5209-5212, 2003. 